\title{
Application of the fruit fly optimization algorithm to an optimized neural network model in radar target recognition
}

\author{
M. Liu $^{1}$, Z.H. Sun ${ }^{2}$ \\ ${ }^{1}$ Sichuan Vocational College of Chemical Technology, Luzhou, Sichuan 646005, China, \\ ${ }^{2}$ Air Force Logistics University, Xuzhou, Jiangsu 221000, China
}

\begin{abstract}
With the development of computer technology, there are more and more algorithms and models for data processing and analysis, which brings a new direction to radar target recognition. This study mainly analyzed the recognition of high resolution range profile (HRRP) in radar target recognition and applied the generalized regression neural network (GRNN) model for HRRP recognition. In order to improve the performance of HRRP, the fruit fly optimization algorithm (FOA) algorithm was improved to optimize the parameters of the GRNN model. Simulation experiments were carried out on three types of aircraft. The improved FOA-GRNN (IFOA-GRNN) model was compared with the radial basis function (RBF) and GRNN models. The results showed that the IFOA-GRNN model had a better convergence accuracy, the highest average recognition rate $(96.4 \%)$, the shortest average calculation time $(275 \mathrm{~s})$, and a good recognition rate under noise interference. The experimental results show that the IFOA-GRNN model has a good performance in radar target recognition and can be further promoted and applied in practice.

Keywords: radar technology, target recognition, generalized regression neural network, highresolution range profile, fruit fly optimization algorithm.

Citation: Liu M, Sun ZH. Application of the fruit fly optimization algorithm to an optimized neural network model in radar target recognition. Computer Optics 2021; 45(2): 296-300. DOI: 10.18287/2412-6179-CO-789.
\end{abstract}

\section{Introduction}

Radar technology can detect and locate long-range targets. It is a vital detection tool and has been applied in both civil and military fields [1], especially in military. With the development of technology, the simple detection and location of targets has not been able to meet the current needs; therefore radar technology has developed towards the recognition of target attributes and categories. How to realize the rapid and accurate identification of targets has become an important development direction of radar technology and has been widely concerned by researchers. Based on various intelligent algorithms, radar target recognition has been extensively and deeply studied. Kang et al. [2] studied Synthetic Aperture Radar (SAR) target recognition, designed an algorithm based on stack automatic encoder, combined unsupervised learning with greedy hierarchical training, and finally analyzed the target using softmax classifier. They found that the method had a classification accuracy of $95.43 \%$. Karine et al. [3] described radar images through statistical modeling, identified targets using use weighted sparse representation method, and found through the experiment that the recognition rate of the method was high. Xiong et al. [4] designed a target recognition method based on multiscale coefficient coding, identified five kinds of ground vehicles, and compared it with other methods through a large number of experiments to verify the effectiveness of the method. Lee [5] studied radar target recognition based on K-nearest neighbor. He extended K-NN rule to realize regression and then carried out target recognition. The re- sult showed that the method was efficient and accurate. In radar target recognition, according to different signals, it can be divided into narrowband and wideband. Wideband signal with high range resolution has a more widely application in target recognition. This paper mainly studied the target recognition method of high resolution range profile (HRRP), designed an improved neural network model: improved fruit fly optimization algorithmGeneralized Regression Neural Network (IFOA-GRNN), and compared it with Radial Basis Function (RBF) and GRNN through simulation experiments to understand the target recognition performance of IFOA-GRNN. This study makes some contributions to the further development of radar technology.

\section{HRRP analysis \\ 1.1. Characteristics of HRRP}

Wideband signals include HRRP [6] and SAR/ISAR image [7]. SAR/ISAR image is a two-dimensional image with relatively high resolution, which can provide many distribution information of target scattering point and is beneficial to the discrimination of complex targets. SAR is generally used for ground targets, while ISAR is usually used for sea or air targets. Their disadvantages are that the processing of the two-dimensional images needs a long time and the recognition is relatively difficult. Compared with the SAR/ISAR image, HRRP has the following characteristics:

(1) it is easier to obtain target HRRP;

(2) HRRP is a one-dimensional vector, which is relatively simple to calculate and requires small storage space; 
(3) target HRRP can give the position information in different directions.

\subsection{Preprocessing}

HRRP refers to the distribution of cross-sectional area of radar of target scatterer (nose, wing, etc.) along the radar line of sight under a certain radar perspective, which can not only reflect the geometric information of the target but also reflect some other characteristic information. The original HRRP data has some problems in intensity and translation sensitivity [8]. To obtain a better target recognition effect, it is necessary to preprocess the HRRP data, mainly including absolute alignment and normalization.

(1) Correlation alignment method

The correlation alignment method refers to moving the range profile to the best position according to certain optimal criteria. The HRRP of radar is set as $X=\left[x_{1}, x_{2}, \ldots, x_{n}\right]^{T}$. For $x(n)$, its Fourier transform is $X(\delta)$. After translation, there is $X^{\prime}(\delta)=X(\delta) e^{-i n t}$. The power spectrum characteristic can be written as follows: $F(\delta)=|X(\delta)|^{2}$

\section{(2) 2-norm normalization}

For $x(n)$, due to amplitude sensitivity, any positive multiple $k x(n)(k>0)$ can also represent the range profile, which can be improved by normalization. The normalized representation of $x(n)$ can be written as follows:

$$
\bar{x}(n)_{\text {energy }}=\frac{x(n)}{\|x(n)\|_{2}}
$$

where $\|x(n)\|_{2}$ stands for 2-norm, i.e., energy.

\section{Improved neural network model 2.1. GRNN model}

GRNN, a kind of neural network [9] proposed by Specht et al. in 1991, is a mathematical model for processing information. It has excellent nonlinear mapping ability and fast learning speed. Its structure is shown in fig. 1.

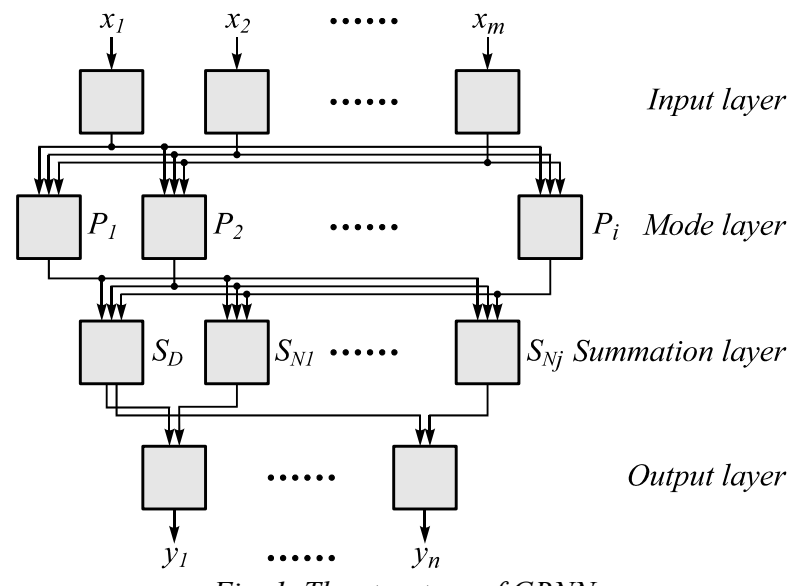

Fig. 1. The structure of GRNN

(1) Input layer: the input of GRNN is $X=\left[x_{1}, x_{2}, \ldots, x_{m}\right]$

(2) Mode layer: the transfer function of the neuron is:

$$
P_{i}=\exp \left[-\frac{\left(X-X_{i}\right)^{T}\left(X-X_{i}\right)}{2 \sigma^{2}}\right],
$$

$i=1,2, \ldots, n$, where $X_{i}$ stands for the learning sample corresponding to the $i$-th neuron and $\sigma$ stands for the smoothing factor, i.e., the width coefficient of Gaussian function. The output of the neuron $i$ is the exponential form of Euclid distance square $D_{i}^{2}=\left(X-X_{i}\right)^{T}\left(X-X_{i}\right)$ between $X$ and $X_{i}$ ( $D$ is the Euclid distance between $X$ and $X_{i}$ ).

(3) Summation layer: the summation layer is composed of two kinds of neurons, and their transfer functions are:

1) $S_{D}=\sum_{i=1}^{n} P_{i}$,

2) $S_{N j}=\sum_{i=1}^{n} y_{i j} P_{i}$

(4) Output layer: the output of GRNN is $Y=\left[y_{1}, y_{2}, \ldots, y_{n}\right]$, one result in the summation layer divides the other result, i.e.,

$$
y_{i}=\frac{S_{N j}}{S_{D}} .
$$

\subsection{Theoretical basis of GRNN}

Suppose that $x$ and $y$ are random variables, the joint probability density function is $f(x, y)$, and the observed value of $x$ is $x^{\prime}$, then the regression value of $y$ about $x^{\prime}$ is:

$$
\hat{y}\left(x^{\prime}\right)=\frac{\int_{-\infty}^{\infty} y f\left(x^{\prime}, y\right) d y}{\int_{-\infty}^{\infty} f\left(x^{\prime}, y\right) d y} .
$$

Then the Parzen nonparametric estimate of the unknown probability density function $f\left(x^{\prime}, y\right)$ is:

$$
f\left(x^{\prime}, y\right)=\frac{\sum_{i=1}^{n} e^{-\frac{\left(x^{\prime}-x_{i}\right)^{T}\left(x^{\prime}-x_{i}\right)}{2 \sigma^{2}}} e^{-\frac{\left(x^{\prime}-y_{i}\right)^{2}}{2 \sigma^{2}}}}{n(2 \pi)^{(a+1) / 2} \sigma^{z+1}} .
$$

The following equation is obtained after integral computation:

$$
\hat{y}\left(x^{\prime}\right)=\sum_{i=1}^{n} y_{i} e\left[-\frac{D^{2}}{2 \sigma^{2}}\right] \div \sum_{i=1}^{n} e\left[-\frac{D^{2}}{2 \sigma^{2}}\right] .
$$

In GRNN, the results are mainly affected by $\sigma$, and its value determines the precision of the network. Therefore, to obtain ideal results, appropriate $\sigma$ value is needed. This study improved the GRNN algorithm by optimizing $\sigma$ value with the improved FOA algorithm.

\subsection{Improved FOA}

FOA, a swarm intelligent algorithm, is a simulation of fruit fly behavior [10]. It has good applications in extremum solution, global optimization, and parameter optimization. 
Therefore, it is used for optimizing the parameters of neural network in this study. The flow of FOA is as follows.

(1) Algorithm initialization: the initial position of the population is set as $X_{0}, Y_{0}$, the population size is set as Sizepop, and the total times of iterations is Maxgen.

(2) Smell search: it is a process of fruit fly making a preliminary determination of the food location by smell and changing the flying direction and distance:

$$
\left\{\begin{array}{l}
X_{i}=X_{0}+\text { random value, } \\
Y_{i}=Y_{0}+\text { random } \text { value }
\end{array}\right.
$$

where $i$ refers to the $i$-th fruit fly and random value is the search radius.

(3) $S_{i}$, the smell concentration discriminant value of the $i$-th fruit fly, i.e., $\sigma$ value of GRNN to be optimized, is calculated:

$$
\left\{\begin{array}{l}
\text { Dist }_{i}=\sqrt{X_{i}^{2}+Y_{i}^{2}}, \\
S_{i}=\frac{1}{\text { Dist }_{i}},
\end{array}\right.
$$

where Dist $_{i}$ represents the distance between the coordinates of fruit fly $i$ and the origin.

(4) Smell $_{i}$, the smell concentration of the position where the fruit fly locates, is calculated: $\operatorname{Smell}_{i}=$ Function $\left(S_{i}\right)$.

(5) The optimal individual in the population is found out: [bestmell bestindex] $=\max \left(\right.$ Smell $\left._{i}\right)$, representing the fruit fly corresponding to the optimal smell concentration.

(6) Visual location: fruit fly can further locate the position of population and food according to vision:

$$
\left\{\begin{array}{l}
\text { smellbest }=\text { bestsmell, } \\
X_{0}=X(\text { bestindex }), \\
Y_{0}=Y(\text { bestindex }) .
\end{array}\right.
$$

(7) Step $(2-5)$ are repeated until the maximum number of iterations is reached.

In FOA, the final $\sigma$ value is related to Dist $_{i}$. In order to avoid FOA falling into local optimum, a method based on individual extremum is adopted to improve FOA, i.e., IFOA. The improvement method is as follows.

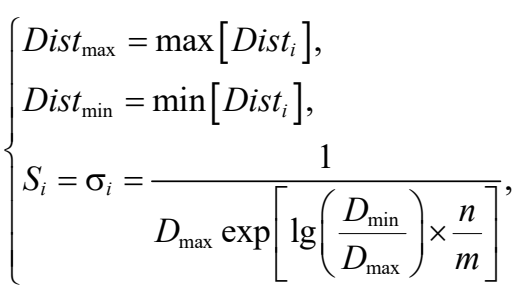

where $n$ is the current number of iterations and $m$ is the maximum number of iterations.

\subsection{Target recognition based on improved neural network}

The target recognition process of IFOA-GRNN is shown in fig. 2.

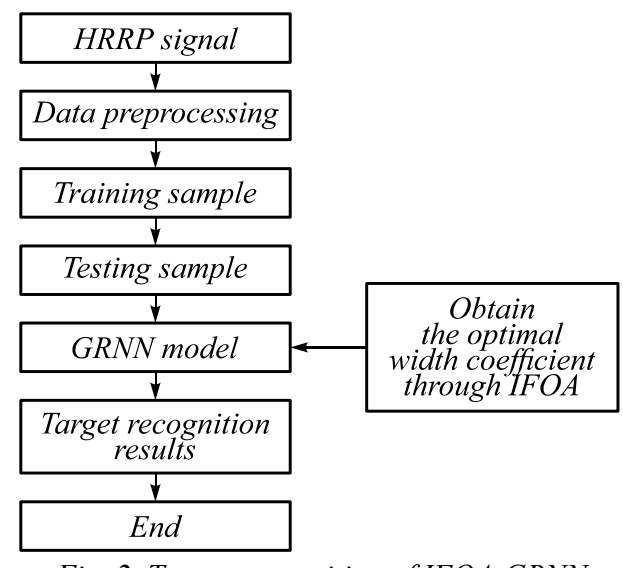

Fig. 2. Target recognition of IFOA-GRNN

As shown in fig. 2, in the radar target recognition by IFOA-GRNN, HRRP is preprocessed by absolute alignment and normalization and then divided into training samples and test samples. The GRNN model is initialized. To further enhance the performance of GRNN, the optimal value of $\sigma$ is obtained through IFOA and substituted into the GRNN model to recognize HRRP, and the recognition result is output.

\section{Simulation experiment 3.1. Experimental data}

The experimental environment was windows7 operating system, $4 \mathrm{~GB}$ memory, $320 \mathrm{~GB}$ disk, and Intel(R)Pentium(R)G630 CPU. The simulation experiment was carried out on the MATLAB platform. The population size of IFOA-GRNN was set as 30, the times of iteration was set as 50 , and the population position interval was $[0,10]$. Targets which needed to be recognized were three types of aircraft, and the parameters are shown in Table 1.

Table 1. Aircraft parameters

\begin{tabular}{|c|l|l|l|}
\hline Model & \multicolumn{1}{|c|}{ A } & \multicolumn{1}{c|}{ B } & \multicolumn{1}{c|}{ C } \\
\hline Length $/ \mathrm{m}$ & 23.6 & 14.5 & 36.6 \\
\hline Width $/ \mathrm{m}$ & 29.1 & 15.8 & 34.5 \\
\hline Height $/ \mathrm{m}$ & 8.9 & 4.6 & 9.6 \\
\hline Radar bandwidth $/ \mathrm{MHz}$ & 400 & 400 & 400 \\
\hline
\end{tabular}

Every aircraft flew with an attitude angle range of $0-$ $180^{\circ}$, and a range profile was recorded every other $1^{\circ}$. The odd attitude angles were taken as the training samples, while the even attitude angles were taken as the testing samples. The HRRP examples of the three kinds of aircraft are shown in figs. 3 - 5. The output node of GRNN was set as 3, which were 001(A), 010(B) and $100(\mathrm{C})$, respectively. RBF [11], GRNN [12] and IFOAGRNN were respectively used for target recognition.

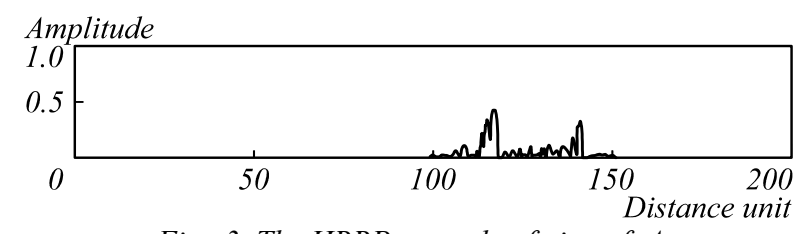

Fig. 3. The HRRP example of aircraft $A$ 


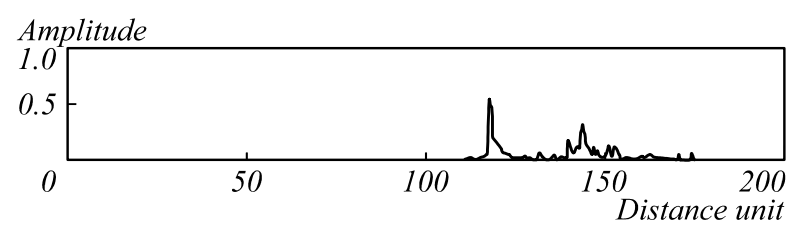

Fig. 4. The HRRP example of aircraft $B$

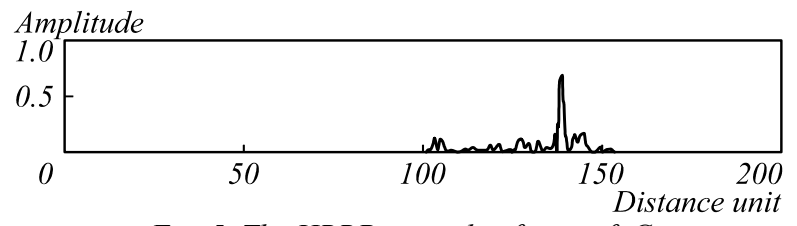

Fig. 5. The HRRP example of aircraft $C$

\subsection{Experimental results}

The training results of the three algorithms are shown in fig. 6 .

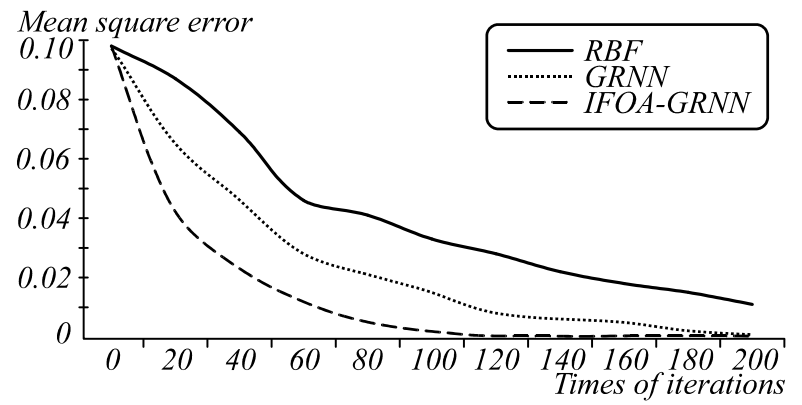

Fig. 6. Comparison of training errors between algorithms

It was seen from fig. 6 that the curve of IFOA-GRNN tended to be stable faster than RBF and GRNN; when the times of iterations were the same, the mean square error of RBF was the largest, followed by GRNN and IFOAGRNN; when the mean square error was the same, the times of iterations of RBF was the smallest, followed by GRNN and IFOA-GRNN. The results showed that the IFOA-GRNN designed in this study had higher convergence accuracy.

The recognition results of the three methods are shown in fig. 7.

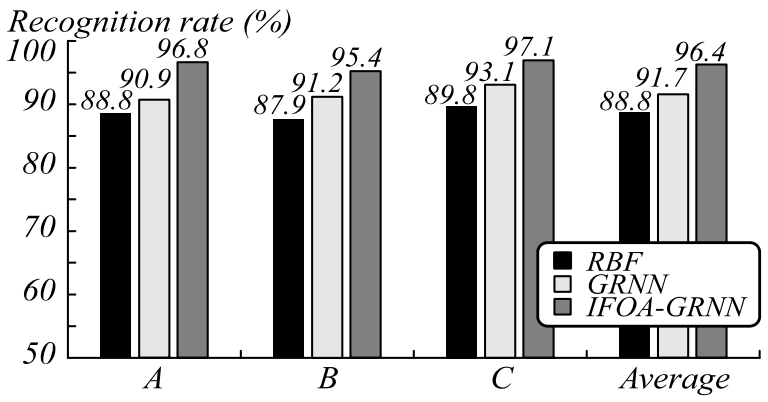

Fig. 7. Comparison of recognition results

It was seen from fig. 7 that the recognition rate of RBF was the lowest, followed by GRNN and IFOAGRNN. In the recognition of aircraft $A$, the recognition rate of IFOA-GRNN was $9 \%$ higher than that of RBF and $6.49 \%$ higher than that of GRNN; in the recognition of aircraft $\mathrm{B}$, the recognition rate of IFOA-GRNN was $8.53 \%$ higher than that of RBF and $4.6 \%$ higher than that of GRNN; in the recognition of aircraft $\mathrm{C}$, the recognition rate of IFOA-GRNN was $8.13 \%$ higher than that of RBF and $4.3 \%$ higher than that of GRNN. The average recognition rate of RBF, GRNN and IFOA-GRNN were $88.8 \%$, $91.7 \%$ and $96.4 \%$ respectively. The above results indicated that the IFOA-GRNN designed in this study had excellent performance in radar target recognition.

The calculation time of the three methods was compared, and the results are shown in Table 2.

Table 2. Comparison of calculation time

\begin{tabular}{|l|c|c|c|}
\hline & RBF & GRNN & IFOA-GRNN \\
\hline Calculation time/s & 978 & 672 & 275 \\
\hline
\end{tabular}

It was seen from Table 2 that the calculation time of RBF was the longest, followed by GRNN and IFOAGRNN, and the calculation time of IFOA-GRNN was $71.88 \%$ and $59.08 \%$ shorter compared with RBF and GRNN, indicating that IFOA-GRNN not only had higher recognition rate but also had a better computational performance.

Radar target recognition is often interfered by noise. In order to verify the anti-jamming ability of the algorithm, $5 \mathrm{~dB}$ and $10 \mathrm{~dB}$ Gaussian white noise were added to HRRP data to analyze the recognition effect of different methods. The results are shown in Table 3.

Table 3. The average recognition rate/\%

\begin{tabular}{|c|c|c|c|}
\hline & RBF & GRNN & IFOA-GRNN \\
\hline $5 \mathrm{~dB}$ & 78.6 & 81.2 & 83.4 \\
\hline $10 \mathrm{~dB}$ & 81.4 & 86.7 & 89.7 \\
\hline
\end{tabular}

It was seen from Table 3 that the radar target recognition effect becomes worse under the noise interference, and the higher the signal-to-noise ratio was, the lower the recognition rate was. However, the recognition rate of IFOA-GRNN was always higher than that of the other two methods. When the noise was $5 \mathrm{~dB}$, the recognition rate of IFOA-GRNN was $6.1 \%$ higher than RBF and $2.2 \%$ higher than GRNN; when the noise was $10 \mathrm{~dB}$, the recognition rate of IFOA-GRNN was $6.5 \%$ higher than that of RBF and $3.46 \%$ higher than GRNN.

\section{Discussion}

Neural network is a simulation of human brain. The simulation in computers can be realized by establishing neural network models. In information processing, neural network has characteristics of strong fault tolerance and superior adaptive ability; therefore it has been widely concerned [13]. It has favourable applications in fields such as computer [14], medicine [15], industry [16] and economy [17] and has a favourable performance in solving the problem of target recognition.

In this study, GRNN was analyzed and improved by IFOA. Then the radar target recognition was realized by the improved neural network. Compared with RBF and GRNN, IFOA-GRNN had better convergence accuracy, could achieve the set mean square error through a small number of iterations, and had better target recognition 
performance. IFOA-GRNN had a higher recognition rate regardless of the type of aircraft. The average recognition rates of the three methods were $88.8 \%, 91.7 \%$ and $96.4 \%$ respectively. The calculation time of RBF, GRNN and IFOA-GRNN was $978 \mathrm{~s}, 672 \mathrm{~s}$ and $275 \mathrm{~s}$ respectively, i.e., the calculation time of IFOA-GRNN was $71.88 \%$ shorter than RBF, indicating that IFOA-GRNN had the highest calculation efficiency. In the aspect of the antijamming ability, the radar target recognition performance obviously declined under the influence of noise, but IFOA-GRNN was less affected by noise and had stronger anti-jamming performance. GRNN is an improvement of RBF. After optimization by IFOA, the performance of the algorithm was further improved; therefore it showed a favourable performance in radar target recognition.

Although some achievements have been made in the optimization of radar target recognition in this study, there are still many deficiencies which need to be improved in the future work:

(1) more optimization methods for neural network model should be searched;

(2) the target recognition performance under the influence of noise should be studied deeply.

\section{Conclusion}

In order to realize the rapid and accurate recognition of radar target, this study designed an improved neural network model: IFOA-GRNN, and compared it with RBF and GRNN through simulation experiment. The results showed that:

(1) the convergence accuracy of RBF was the lowest, followed by GRNN and IFOA-GRNN;

(2) the average recognition rate of RBF, GRNN and IFOA-GRNN was $88.8 \%, 91.7 \%$ and $96.4 \%$ respectively;

(3) the average calculation time of RBF, GRNN and IFOA-GRN was 978 s, 672 s and 275 s, respectively;

(4) the recognition rate of IFOA-GRNN was significantly higher in the case of noise interference.

The experimental results show that the IFOA-GRNN model is reliable in the radar target recognition, which is worth further promotion and application in practice to improve radar target recognition technology.

\section{References}

[1] Shu T, Tang B, Yin K, Sun Q, Chen Y, Yu W. Development of multichannel real-time Hardware-in-the-Loop ra- dar environment simulator for missile-borne Synthetic Aperture Radar. Biochem J 2015; 40(2): 368-373.

[2] Kang M, Ji K, Leng X, Xing X, Zou H. Synthetic aperture radar target recognition with feature fusion based on a stacked autoencoder. Sensors 2017; 17(1): 192.

[3] Karine A, Toumi A, Khenchaf A, Hassouni ME. Target recognition in radar images using weighted statistical dictionary-based sparse representation. IEEE Geosci Remote Sens Lett 2017; 14(12): 2403-2407.

[4] Xiong W, Zhang G, Liu S, Yin J. Multiscale kernel sparse coding-based classifier for HRRP radar target recognition. IET Radar Sonar Navig 2016; 10(9): 1594-1602.

[5] Lee KC. Radar target recognition by machine learning of $\mathrm{K}$-nearest neighbors regression on angular diversity RCS. Appl Comput Electromagn Soc J 2019; 34(1): 75-81.

[6] Feng B, Chen B, Liu H. Radar HRRP target recognition with deep networks. Patt Recogn 2017; 61(Complete): 379-393.

[7] Karine A, Toumi A, Khenchaf A, Hassouni ME. Radar target recognition using salient keypoint descriptors and multitask sparse representation. Remote Sens 2018; 10(6): 843.

[8] Guo Y, Xiao H, Kan Y, Fu Q. Learning using privileged information for HRRP-based radar target recognition. IET Signal Process 2018; 12(2): 188-197.

[9] Specht DF. A general regression neural network. IEEE Trans Neural Netw 1991; 2(6): 568-576.

[10] Xing B, Gao W-J. Fruit fly optimization algorithm. In Book: Xing B, Gao W-J. Innovative computational intelligence: A rough guide to 134 clever algorithms. Switzerland: Springer International Publishing; 2014: 167-170.

[11] Cecati C, Kolbusz J, Rozycki P, Siano P, Wilamowski BM. A Novel RBF training algorithm for short-term electric load forecasting and comparative studies. IEEE Trans Ind Electron 2015; 62(10): 6519-6529.

[12] Pierre OC. GRNN: General regression neural network. Revue de Physique Appliquée 2013, iv(6): 1321-1325.

[13] Wang F, Devabhaktuni VK, Xi C, Zhang Q. Neural network structures and training algorithms for RF and microwave applications. Int J RF Microw C E 2015; 9(3): 216-240.

[14] Chi P, Li S, Xu C, Zhang T, Zhao J, Liu Y, Wang Y, Xie Y. PRIME: A Novel processing-in-memory architecture for neural network computation in ReRAM-based main memory. Computer Architecture News 2016; 44(3): 27-39.

[15] Moeskops P, Viergever MA, Mendrik AM, de Vries LS, Benders MJNL, Išgum I. Automatic segmentation of MR brain images with a convolutional neural network. IEEE Trans Med Imaging 2017; 35(5): 1252-1261.

[16] Ben Ali J, Fnaiech N, Saidi L, Chebel-Morello B, Fnaiech F. Application of empirical mode decomposition and artificial neural network for automatic bearing fault diagnosis based on vibration signals. Appl Acoust 2015; 89: 16-27.

[17] Yu Y, Wang YR, Gao SC, Zheng T. Statistical modeling and prediction for tourism economy using dendritic neural network. Comput Intell Neurosci 2017; 2017: 1-9.

\section{Authors' information}

Min Liu (b. 1982) received the bachelor's degree from Sichuan Normal University in 2005. She is a lecturer in Sichuan Vocational College of Chemical Technology, China. She is interested in computer science and technology. E-mail: $\underline{11 u 801 @ 126 . c o m . ~}$

Zhihong Sun (b. 1978) received the master's degree from Beijing Institute of Technology in 2007. She is a lecturer in Air Force Logistics University, China. She is interested in higher mathematics education and algorithm.

E-mail:szr6e5@yeah.net.

Received July 27, 2020. The final version - January 19, 2021. 